\title{
Coupling architecture between INS/GPS for precise navigation on set paths
}

\author{
Autores \\ Jesús Silva, Noel Varela, Omar Bonerge Pineda Lezama, Hugo Hernández Palma, \\ Eduardo Nicolas Cueto
}

\begin{abstract}
GPS offers the advantage of providing high long-term position accuracy with residual errors that affect the final positioning solution to a few meters with a sampling frequency of $1 \mathrm{~Hz}$ (Marston et al. in Decis Support Syst 51:176-189, 2011 [1]]). The signals are also subject to obstruction and interference, so GPS receivers cannot be relied upon for a continuous navigation solution. On the contrary, the inertial navigation system has a sampling frequency of at least $50 \mathrm{~Hz}$ and exhibits low noise in the short term. In this research, a prototype based on development cards is implemented for the coupling of the inertial navigation system with GPS to improve the precision of navigation on a trajectory.
\end{abstract}

Palabras clave

Global positioning system (GPS), Inertial measurement unit, Coupling system, Sensors, Kalman filter, Madgwick filter. 